\title{
PERSPECTIVA DEL DERECHO DEL MEDIO AMBIENTE Y DE LAS POLÍTICAS AMBIENTALES DE LA UNIÓN EUROPEA
}

\author{
JORGE AGUdo GONZÁLEZ \\ Profesor titular de Derecho Administrativo \\ Universidad Autónoma de Madrid \\ LORENA TRUJILlo PARRA \\ Doctoranda \\ Universidad Autónoma de Madrid
}


Sumario: 1. Introducción. 2. Actividad de las instituciones en el ámbito de la política europea de medio ambiente. 2.1. Actos conjuntos del Consejo y del Parlamento Europeo. A. Directivas. B. Reglamentos. 2.2. Actos del Consejo. A. Reglamentos. B. Decisiones. 2.3. Actos de Parlamento. 2.4. Actos de la Comisión. A. Reglamentos. B. Reglamentos de ejecución. C. Reglamentos delegados. D. Decisiones de Ejecución. E. Dictámenes y comunicaciones de la Comisión. 2.5. Otros. A. Comité de las Regiones. B. Comité Económico y Social Europeo.

\section{Introducción}

Como viene siendo habitual, la Unión Europea ha dictado numerosos actos en el marco de su política medioambiental. No obstante, estos han descendido en número en los últimos años. Dentro de los actos jurídicos dictados en la materia, cada vez gozan de mayor protagonismo, al menos cuantitativamente, los dictados por la Comisión, especialmente los actos de ejecución y modificación de normas aprobadas con anterioridad. Igualmente, se deben recalcar los actos jurídicos no vinculantes, destacando principalmente los dictámenes del Comité Económico y Social y del Comité de las Regiones. Desde un punto de vista estrictamente material, destacan los actos en materia de pesca.

La exposición, como venimos realizando, se ordenará en función de la institución emisora del acto jurídico, y, dentro de la actividad de cada institución, se atenderá, en la medida de lo posible, a una exposición temática por materias.

\section{Actividad de las instituciones en el ámbito de la política europea de medio ambiente}

\subsection{Actos conjuntos del Consejo y del Parlamento Europeo}

\section{A. Directivas}

La Decisión núm. 1386/2013/UE del Parlamento Europeo y del Consejo reconoce que las emisiones de agentes contaminantes del aire se han reducido considerablemente en las últimas décadas, pero, al mismo tiempo, señala que los niveles de contaminación atmosférica siguen siendo problemáticos en muchas partes de Europa y que los ciudadanos de la Unión continúan estando expuestos a contaminantes atmosféricos que pueden poner en peligro su salud y bienestar. Lamentablemente, los niveles de contaminación atmosférica en muchas zonas de la Unión siguen estando por encima de los límites fijados por la Unión y las normas de calidad del aire de la Unión siguen incumpliendo los objetivos establecidos por la Organización Mundial de la Salud. 
En este sentido, el Programa de Medio Ambiente aboga por que las medidas que se adopten a nivel local se complementen con una política adecuada, tanto a nivel nacional como de la Unión, y que se intensifiquen los esfuerzos para cumplir plenamente la legislación sobre calidad del aire de la Unión y que se determinen acciones y objetivos estratégicos para después de 2020.

Las emisiones de contaminantes procedentes de la combustión de combustibles en las instalaciones de combustión medianas no están en general reguladas a nivel de la Unión, a pesar de que contribuyen cada vez más a la contaminación del aire, debido en particular al creciente uso de la biomasa como combustible, impulsado por las políticas sobre clima y energía. Por todo ello, se dicta la Directiva (UE) 2015/2193 del Parlamento Europeo y del Consejo, de 25 de noviembre de 2015, sobre la limitación de las emisiones a la atmósfera de determinados agentes contaminantes procedentes de las instalaciones de combustión medianas. En esta directiva se establece que las instalaciones de combustión medianas deben desarrollarse y explotarse de modo que se fomente la eficiencia energética mediante su modernización, incluyendo consideraciones de índole económica, las posibilidades técnicas y el ciclo de vida de dichas instalaciones.

La Directiva debe aplicarse a las instalaciones de combustión, incluidas las combinaciones formadas por dos o más nuevas instalaciones de combustión medianas, cuya potencia térmica nominal sea igual o superior a $1 \mathrm{MW}$ e inferior a $50 \mathrm{MW}$. Las instalaciones de combustión individuales cuya potencia térmica nominal sea inferior a 1 MW no deben tenerse en cuenta a efectos del cálculo de la potencia térmica nominal total de una combinación de instalaciones de combustión. Con el fin de evitar una laguna normativa, las disposiciones de la Directiva deben aplicarse asimismo a las combinaciones formadas por nuevas instalaciones de combustión medianas cuya potencia térmica nominal total sea igual o superior a $50 \mathrm{MW}$, sin perjuicio de lo dispuesto en el capítulo III de la Directiva 2010/75/UE.

Para asegurar el control de las emisiones atmosféricas, la Directiva establece valores límite de emisión y requisitos de seguimiento, indicados en su anexo II. Asimismo, respecto a las emisiones de dióxido de azufre, óxidos de nitrógeno y partículas, una instalación de combustión mediana solo debe funcionar si se le ha concedido un permiso o si ha sido registrada por la autoridad competente sobre la base de la información facilitada por su titular. 
Por último, y con el fin de disponer de tiempo suficiente para adaptar técnicamente las instalaciones de combustión medianas existentes a los requisitos de la Directiva, los valores límite de emisión se aplicarán a esas instalaciones tras un plazo fijado a partir de la fecha de aplicación de la Directiva.

\section{B. Reglamentos}

El Acuerdo para el establecimiento de la Comisión General de Pesca del Mediterráneo ("el Acuerdo CGPM") dispone un marco adecuado para la cooperación multilateral con el fin de promover el desarrollo, la conservación, la gestión racional y el mejor aprovechamiento de los recursos marinos vivos en el Mediterráneo y en el mar Negro en niveles considerados sostenibles y que presenten un bajo riesgo de agotamiento. Esta comisión adopta regularmente medidas de gestión sobre la explotación de los recursos en el Mediterráneo, medidas para la reducción de las capturas accidentales de aves marinas, tortugas marinas, focas monje y cetáceos en las actividades pesqueras, y medidas relativas a la utilización de determinadas tecnologías en la pesca, tales como la restricción de la utilización de vehículos submarinos teledirigidos, entre otras. Procede, por lo tanto, incluir todas estas medidas y recomendaciones en los instrumentos jurídicos pertinentes.

En este sentido, con el fin de mejorar la recogida de datos con vistas al seguimiento científico de determinadas especies marinas capturadas accidentalmente en las artes de pesca, los capitanes de los buques pesqueros estarán obligados a registrar las capturas accidentales de las especies marinas afectadas. Los informes nacionales destinados al Comité Asesor Científico de la CGPM deberán contener la información proporcionada por los buques de pesca sobre capturas accidentales de determinadas especies marinas, acompañada de datos pormenorizados procedentes de fuentes disponibles sobre estos incidentes. Asimismo, a fin de garantizar condiciones uniformes de ejecución de determinadas disposiciones del presente reglamento, se conferirán a la Comisión competencias de ejecución en lo que respecta al formato de presentación y a la transmisión de datos sobre extracción de coral rojo y a la información sobre las capturas accidentales de aves marinas, tortugas marinas, focas monje, cetáceos, tiburones y rayas; las modificaciones de las listas de los puertos designados para el desembarque del coral rojo extraído; las repercusiones de determinados buques pesqueros sobre las poblaciones de cetáceos; y los cambios producidos en los mapas y las listas de 
posiciones geográficas que identifican el emplazamiento de las cuevas de focas monje. Además, se delegarán en la Comisión los poderes para adoptar actos, con arreglo al artículo 290 del Tratado, en lo que respecta a las autorizaciones para establecer las excepciones que estime pertinentes, conforme a los informes científicos del momento. Por todo ello, se adopta el Reglamento (UE) 2015/2102 del Parlamento Europeo y del Consejo, de 28 de octubre de 2015, por el que se modifica el Reglamento (UE) núm. 1343/2011 sobre determinadas disposiciones aplicables a la pesca en la zona del Acuerdo CGPM (Comisión General de Pesca del Mediterráneo).

\subsection{Actos del Consejo}

\section{A. Reglamentos}

El artículo 43, apartado 3. ${ }^{\circ}$, del Tratado de Funcionamiento de la Unión Europea establece que el Consejo, a propuesta de la Comisión, adoptará las medidas relativas a la fijación y el reparto de posibilidades de pesca tanto en aguas de la Unión Europea como en aguas internacionales, cuando existan acuerdos, así como determinadas condiciones relacionadas funcionalmente con ellas, cuando proceda.

En el ámbito de las aguas internacionales, la Unión Europea ha rubricado nuevos protocolos de los acuerdos de asociación con diferentes Estados, y ha concedido a los buques de la Unión posibilidades de pesca en zonas de pesca bajo la soberanía o la jurisdicción de otros Estados, pero, tal y como se desprende del derecho de la Unión, conviene determinar el método de reparto de las posibilidades de pesca entre los Estados miembros durante el período de aplicación de los protocolos. A tales efectos, tal y como se desprende de las obligaciones del Consejo en esta materia, se dictan los siguientes reglamentos:

- Reglamento (UE) 2015/2192 del Consejo, de 10 de noviembre de 2015, relativo al reparto de las posibilidades de pesca en virtud del Protocolo por el que se fijan las posibilidades de pesca y la contrapartida financiera previstas en el Acuerdo de Asociación en el sector pesquero entre la Comunidad Europea y la República Islámica de Mauritania por un período de cuatro años.

- Reglamento (UE) 2015/2313 del Consejo, de 30 de noviembre de 2015, relativo a la asignación de las posibilidades de pesca en virtud del Protocolo de aplicación del 
Acuerdo de colaboración de pesca sostenible entre la Unión Europea y la República de Liberia.

Pasando a otra cuestión, en el ámbito de las aguas de la Unión Europea las posibilidades de pesca han de asignarse por el Consejo a los Estados miembros de tal modo que se garantice la estabilidad relativa de las actividades pesqueras de cada uno de ellos en relación con cada población o pesquería y teniendo debidamente en cuenta los objetivos de la política pesquera común establecidos en el Reglamento (UE) núm. 1380/2013. De conformidad con el Reglamento (UE) núm. 1380/2013, los totales admisibles de capturas deben establecerse sobre la base de los dictámenes científicos disponibles, teniendo en cuenta los aspectos biológicos y socioeconómicos, asegurando al mismo tiempo un trato justo a los distintos sectores de la pesca y en función de las opiniones expresadas durante la consulta a los interesados. En este sentido, se dicta el Reglamento (UE) 2015/1961 del Consejo, de 26 de octubre de 2015, por el que se modifica el Reglamento (UE) 2015/104 en lo que respecta a determinadas posibilidades de pesca, que, atendiendo a los dictámenes de los científicos del Consejo Internacional para la Exploración del Mar, limita la captura del arenque, indican un aumento significativo en la población de la solla, pudiendo aumentar su cuota total admisible de captura, y permite un total de capturas deseadas de 506.000 toneladas de espadín para el periodo comprendido entre junio de 2015 y 2016.

Asimismo, debido a cambios en la biología de la población oriental de bacalao, el Consejo Internacional para la Exploración del Mar considera que se deben fijar y asignar las posibilidades de pesca de bacalao para no poner en grave peligro la sostenibilidad de la población de este pez, por lo que aplicará un planteamiento por etapas en la reducción de sus posibilidades de pesca. En este sentido, se dicta el Reglamento (UE) 2015/2072 del Consejo, de 17 de noviembre de 2015, por el que se establecen, para 2016, las posibilidades de pesca para determinadas poblaciones y grupos de poblaciones de peces aplicables en el mar Báltico y por el que se modifican los Reglamentos (UE) núm. 1221/2014 y (UE) 2015/104.

En esta misma dirección también se han dictado el Reglamento (UE) 2016/73 del Consejo, de 18 enero de 2016, por el que se fijan, para 2016, las posibilidades de pesca de determinadas poblaciones de peces aplicables en el mar Negro, y el Reglamento (UE) 2016/72 del Consejo, de 22 de enero de 2016, por el que se establecen, para 2016, las posibilidades de pesca para determinadas poblaciones y grupos de poblaciones de 
peces, aplicables en aguas de la Unión y, en el caso de los buques pesqueros de la Unión, en determinadas aguas no pertenecientes a la Unión, y por el que se modifica el Reglamento (UE) 2015/104. Respecto al Reglamento (UE) 2016/72, cabe destacar, por una parte, el nuevo método de cálculo para garantizar una compensación justa por capturas previamente descartadas, cuyo desembarque es obligatorio a partir del 1 de enero de 2016. Dicho método es el siguiente: la nueva cifra de desembarques debe calcularse restando a la cifra del total de capturas del Consejo Internacional para la Exploración del Mar las cantidades que seguirán siendo descartadas durante el período de desembarque obligatorio; el aumento ulterior de la cifra del total admisible de capturas deberá ser proporcional a la diferencia entre la nueva cifra de desembarques calculada y la cifra anterior de desembarques del Consejo Internacional para la Exploración del Mar. Por otra, cabe destacar la situación de diversas especies. Así, el dictamen científico sobre la población de lubina del mar Céltico, del canal de la Mancha, del mar de Irlanda y del sur del mar del Norte señala que se encuentra en peligro y que la población sigue disminuyendo. En relación con las poblaciones de elasmobranquios (tiburones y rayas), obedeciendo al deficiente estado de conservación de tales poblaciones, sus capturas continúan estando prohibidas y una disposición establece la obligación de liberar de inmediato las capturas accidentales.

En otro orden de consideraciones, y pasando a un tema distinto, debemos poner de manifiesto que, como consecuencia del accidente ocurrido en la central nuclear de Chernóbil el 26 de abril de 1986, se liberaron a la atmósfera cantidades considerables de materiales radiactivos que contaminaron los alimentos y los piensos de varios países europeos a unos niveles significativos desde el punto de vista de la salud. Se adoptaron determinadas medidas para garantizar que ciertos productos agrícolas solo se introdujesen en la Unión con arreglo a acuerdos comunes que salvaguardasen la salud de la población, conservando al mismo tiempo el carácter unificado del mercado y evitando las desviaciones de los flujos comerciales. En este contexto se adoptó el Reglamento (Euratom) núm. 3954/87 del Consejo, que establece las tolerancias máximas de contaminación radiactiva que deben aplicarse tras un accidente nuclear o cualquier otro caso de emergencia radiológica que pueda producir o haya producido una contaminación radiactiva significativa de los alimentos y los piensos. Estas tolerancias máximas siguen respetando las recomendaciones científicas más recientes disponibles en la actualidad a escala internacional. 
A raíz del accidente ocurrido en la central nuclear de Fukushima el 11 de marzo de 2011, se informó a la Comisión de que los niveles de radionucleidos presentes en algunos productos alimenticios procedentes de Japón superaban los umbrales de intervención aplicables en ese país. Dado que dicha contaminación podía constituir una amenaza para la salud pública y animal de la Unión, se adoptaron medidas que imponían condiciones especiales para la importación de alimentos y piensos producidos en o procedentes de Japón.

Ante tales hechos, es necesario establecer un sistema que permita a la Comunidad, tras un accidente nuclear o cualquier otro caso de emergencia radiológica que pueda producir o haya producido una contaminación radiactiva significativa de los alimentos y de los piensos, fijar tolerancias máximas de contaminación radiactiva para los productos que vayan a comercializarse con el fin de proteger a la población. Para ello, se adopta el Reglamento (Euratom) 2016/52 del Consejo, de 15 de enero de 2016, por el que se establecen tolerancias máximas de contaminación radiactiva de los alimentos y los piensos tras un accidente nuclear o cualquier otro caso de emergencia radiológica, y se derogan el Reglamento (Euratom) núm. 3954/87 del Consejo y los Reglamentos (Euratom) núm. 944/89 y (Euratom) núm. 770/90 de la Comisión. El presente Reglamento debe constituir la lex specialis para el procedimiento de adopción y ulterior modificación de los reglamentos de ejecución por los que se establecen las tolerancias máximas aplicables de contaminación radiactiva tras un caso de emergencia radiológica.

\section{B. Decisiones}

Atendiendo a la caducidad de determinados acuerdos de colaboración que fijaban las posibilidades de pesca y la contrapartida financiera establecidas entre la Unión y otros Estados, el Consejo ha adoptado diversas decisiones mediante las cuales ha autorizado a la Comisión a negociar nuevos protocolos de acuerdos de colaboración por los que se conceden a los buques de la Unión posibilidades de pesca en diversas zonas de otros Estados con el fin de garantizar la continuidad de las actividades pesqueras de los buques de la Unión. Hasta la adopción de los nuevos acuerdos de colaboración se aplicarán los anteriores de forma provisional.

En este sentido se ha adoptado, en primer lugar, la Decisión (UE) 2015/1893 del Consejo, de 5 de octubre de 2015, relativa a la celebración en nombre de la Unión 
Europea, del Protocolo por el que se fijan las posibilidades de pesca y la contrapartida financiera previstas en el Acuerdo de colaboración en el sector pesquero entre la República de Madagascar y la Comunidad Europea. En segundo lugar, la Decisión (UE) 2015/1894 del Consejo, de 5 de octubre de 2015, relativa a la celebración del Protocolo entre la Unión Europea y la República de Cabo Verde por el que se fijan las posibilidades de pesca y la contrapartida financiera previstas en el Acuerdo de Asociación en el sector pesquero entre la Comunidad Europea y la República de Cabo Verde. En tercer lugar, la Decisión (UE) 2015/2103 del Consejo, de 16 de noviembre de 2015, relativa a la firma, en nombre de la Unión Europea, y a la aplicación provisional del Protocolo por el que se fijan las posibilidades de pesca y la contrapartida financiera establecidas en el Acuerdo de colaboración en materia de pesca entre la Comunidad Europea, por una parte, y el Gobierno de Dinamarca y el Gobierno Autónomo de Groenlandia, por otra. En cuarto lugar, la Decisión (UE) 2015/2191 del Consejo, de 10 de noviembre de 2015, relativa a la firma, en nombre de la Unión Europea, y a la aplicación provisional del Protocolo por el que se fijan las posibilidades de pesca y la contrapartida financiera previstas en el Acuerdo de Asociación en el sector pesquero entre la Comunidad Europea y la República Islámica de Mauritania por un período de cuatro años. En quinto y último lugar, la Decisión (UE) 2015/2312 del Consejo, de 30 de noviembre de 2015, relativa a la firma, en nombre de la Unión Europea, y a la aplicación provisional del Acuerdo de colaboración de pesca sostenible entre la Unión Europea y la República de Liberia y de su Protocolo de aplicación.

En relación con esta última decisión, debemos señalar que, a fecha de 12 de diciembre de 2015, se firmó el Acuerdo de Colaboración de Pesca Sostenible entre la Unión Europea y la República de Liberia, con el fin de cooperar, en interés mutuo, para promover el establecimiento de una pesca responsable que garantice la conservación a largo plazo y la explotación sostenible de los recursos biológicos marinos. Este acuerdo establece normas y condiciones que regulan las actividades pesqueras de los buques de la Unión en la zona de pesca de Liberia y el apoyo ofrecido por la Unión para el desarrollo de una pesca sostenible en dicha zona, incluido el desarrollo de contenido local de Liberia.

\subsection{Actos del Parlamento}


Atendiendo a lo dispuesto en el artículo 349 del Tratado de Funcionamiento de la Unión Europea, que establece la necesidad de promover políticas específicas para las regiones ultraperiféricas, en particular, en el sector de la pesca, así como a diversas consideraciones en relación con la situación de la pesca comunitaria en el ámbito de la pesca artesanal y de bajura - es decir, la pesca practicada por buques de menos de 15 metros de eslora-, que supone alrededor del $80 \%$ de la pesca comunitaria, entre otras, se adopta la Resolución del Parlamento Europeo, de 22 de noviembre de 2012, sobre la pesca artesanal y de pequeña escala y la reforma de la política pesquera común $(2011 / 2292(\mathrm{INI}))(2015 / \mathrm{C} 419 / 25)$, la cual establece que la pesca de pequeña escala reviste, por sus características y su peso en el conjunto del sector, una importancia crucial para la consecución de los que deberían ser los objetivos primordiales de cualquier política de pesca, a saber: garantizar el abastecimiento público de pescado para la población y el desarrollo de las comunidades costeras, y promover el empleo y la mejora de las condiciones de vida de los profesionales de la pesca, en un marco que garantice la sostenibilidad y la buena conservación de los recursos.

Dicha Resolución entiende que debe partirse de una definición genérica de la pesca artesanal que evite que la múltiple casuística de la pesca — según las distintas zonas—, el tipo de recursos explotados o cualquier otra particularidad de carácter puramente local pueda tener como consecuencia el incumplimiento de los objetivos de simplificación, claridad normativa y no discriminación; y considera asimismo que la Política Pesquera Común debe incorporar medidas que permitan una cierta flexibilidad en aquellos casos, demostrables científicamente, en que la actividad pesquera no sería posible sin determinadas adaptaciones de las normas generales.

Además, se recalca la necesidad de tener en cuenta los estudios científicos existentes sobre la pesca de pequeña escala; señala que algunos de esos estudios presentan propuestas de definición de la "pesca de pequeña escala", como es el caso del proyecto "PRESPO", que propone un enfoque basado en descriptores numéricos para la definición y segmentación de las flotas europeas de pesca artesanal.

Pasando a otro orden de consideraciones, y entendiendo que la protección de los animales en el siglo XXI es una manifestación de humanidad y un reto para la civilización y la cultura europeas, cualquier acción con vistas a garantizar la protección y el bienestar de los animales debe basarse en descubrimientos científicos y en el principio de que los animales son seres sensibles cuyas necesidades específicas deben 
ser tenidas en cuenta, tal como se establece en el artículo 13 del Tratado de Funcionamiento de la Unión Europea. Partiendo de esa premisa, el transporte de animales es un resultado de factores económicos y logísticos que puede generar a la vez costes sociales y medioambientales adicionales, por lo que se ha de abordar a través de un enfoque intracomunitario, pero también desde una perspectiva extracomunitaria, puesto que los animales procedentes de terceros países han de ser objeto de un control y seguimiento exhaustivos que aseguren un mayor equilibrio de las condiciones de competencia para los productores europeos y supongan un incentivo para la mejora de las condiciones del transporte de animales en terceros países. En este sentido, se adopta la Resolución del Parlamento Europeo, de 12 de diciembre de 2012, sobre la protección de los animales durante el transporte (2012/2031(INI))(2015/C 434/07), por la que se insta a la Comisión que garantice una observancia eficaz y uniforme de la legislación de la UE existente sobre el transporte de animales en todos los Estados miembros. Por otro lado, para garantizar la eficacia y viabilidad de la legislación existente, es fundamental mejorar su observancia a fin de mejorar las condiciones de transporte y evitar las distorsiones de la competencia en los Estados miembros de la UE.

En el marco de la Conferencia de las Partes de la Convención sobre el Comercio Internacional de Especies Amenazadas de Fauna y Flora Silvestres (CITES), que se celebró en Bangkok del 3 al 14 de marzo de 2013, se dictó la Resolución del Parlamento Europeo, de 6 de febrero de 2013, sobre los objetivos estratégicos de la UE para la Decimosexta reunión de la Conferencia de las Partes de la Convención sobre el Comercio Internacional de Especies Amenazadas de Fauna y Flora Silvestres, que se celebrará en Bangkok (Tailandia) del 3 al 14 de marzo de 2013 (2012/2838(RSP))(2016/C 024/04). La CITES es el acuerdo mundial vigente más amplio en materia de conservación de la naturaleza; previene la explotación excesiva por el comercio internacional de especies de fauna y flora silvestres; y la han ratificado las 177 partes de la Convención, incluidos los 27 Estados miembros de la Unión Europea. Su objetivo principal es garantizar que el comercio internacional de animales y plantas salvajes no represente una amenaza para la supervivencia de las especies en estado salvaje.

En este contexto, el Parlamento pide a la Unión Europea y a los Estados miembros que adopten el principio de precaución como principio rector en todas sus decisiones sobre los documentos de trabajo y las propuestas de listas; que tengan igualmente presentes el 
principio de imposición de costes a los usuarios y el enfoque sobre los ecosistemas y los principios tradicionales de conservación; y que asuman un papel relevante en la protección de las especies en peligro de extinción, participando activamente en las negociaciones de la Convención CITES y promoviendo la supresión de las fisuras que agravan esta situación y que lo hagan con una sola voz, para mejorar la celeridad y la eficiencia de sus procedimientos internos de toma de decisiones, a fin de poder consensuar rápidamente una posición interna de la UE con vistas a la COP 16 de la CITES y aprovechar al máximo la fortaleza de tener veintisiete partes dentro de la UE para acercar las decisiones de la Conferencia hacia el principio de precaución.

\subsection{Actos de la Comisión}

\section{A. Reglamentos}

Conforme a lo dispuesto en el artículo 6, apartado 1. ${ }^{\circ}$, del Reglamento (CE) núm. 216/2008 del Parlamento Europeo y del Consejo, de 20 de febrero de 2008, sobre normas comunes en el ámbito de la aviación civil y por el que se crea una Agencia Europea de Seguridad Aérea, los productos, componentes y equipos deben ajustarse a los requisitos de protección medioambiental incluidos en los volúmenes I y II del anexo 16 del Convenio sobre Aviación Civil Internacional. Para ello, se adopta el Reglamento (UE) 2016/4 de la Comisión, de 5 de enero de 2016, por el que se modifica el Reglamento (CE) núm. 216/2008 del Parlamento Europeo y del Consejo en lo que se refiere a los requisitos esenciales de protección medioambiental, y el Reglamento (UE) 2016/5 de la Comisión, de 5 de enero de 2016, por el que se modifica el Reglamento (UE) núm. 748/2012 en lo que se refiere a la aplicación de requisitos esenciales de protección medioambiental.

En materia de residuos de plaguicidas, la Comisión ha consultado con los laboratorios de referencia de la Unión Europea sobre la necesidad de adaptar determinados límites de determinación analítica. Estos laboratorios concluyeron que el progreso técnico permite, para ciertas mercancías, establecer límites más bajos de determinación analítica de diversas sustancias.

En los anexos del Reglamento (CE) núm. 396/2005 se fijaron los límites máximos de estos residuos (LMR). En el marco de un procedimiento de autorización del uso de 
determinados productos fitosanitarios, se presentaron varias solicitudes de modificación de estos límites.

De conformidad con el procedimiento previsto en el Reglamento (CE) núm. 396/2005, los Estados miembros afectados evaluaron estas solicitudes y enviaron los correspondientes informes de evaluación a la Comisión. La Autoridad Europea de Seguridad Alimentaria estudió las solicitudes y los informes de evaluación, prestando especial atención a los riesgos para el consumidor y, en su caso, para los animales, y emitió dictámenes motivados sobre los LMR propuestos y remitió dichos dictámenes a la Comisión y a los Estados miembros y los puso a disposición del público.

De acuerdo con los dictámenes motivados de la Autoridad Europea de Seguridad Alimentaria y de la Organización Mundial del Comercio, una vez consultados los socios comerciales de la Unión y teniendo en cuenta los factores pertinentes para la cuestión objeto de consideración, las modificaciones pertinentes de los LMR cumplen los requisitos del Reglamento (CE) núm. 396/2005. En consecuencia, se admiten las solicitudes presentadas y para ello se adoptan los siguientes reglamentos:

- Reglamento (UE) 2015/1910 de la Comisión, de 21 de octubre de 2015, que modifica los anexos III y V del Reglamento (CE) núm. 396/2005 del Parlamento Europeo y del Consejo por lo que respecta a los límites máximos de residuos de guazatina en determinados productos.

- Reglamento (UE) 2015/2075 de la Comisión, de 18 de noviembre de 2015, que modifica los anexos II y III del Reglamento (CE) núm. 396/2005 del Parlamento Europeo y del Consejo por lo que respecta a los límites máximos de residuos de abamectina, desmedifam, diclorprop-P, haloxifop-P, orizalina $\mathrm{y}$ fenmedifam en determinados productos.

- Reglamento (UE) 2016/1 de la Comisión, de 3 de diciembre de 2015, que modifica los anexos II y III del Reglamento (CE) núm. 396/2005 del Parlamento Europeo y del Consejo en lo relativo a los límites máximos de residuos de bifenazato, boscalid, ciazofamida, ciromazina, dazomet, ditiocarbamatos, fluazifop-P, mepanipirima, metrafenona, picloram, propamocarb, piridabeno, piriofenona, sulfoxaflor, tebuconazol, tebufenpirad y tiram en determinados productos.

- Reglamento (UE) 2016/46 de la Comisión, de 18 de enero de 2016, que modifica el anexo III del Reglamento (CE) núm. 396/2005 del Parlamento Europeo y del Consejo 
por lo que respecta a los límites máximos de residuos de oxadixilo y espinetoram en determinados productos.

- Reglamento (UE) 2016/53 de la Comisión, de 19 de enero de 2016, por el que se modifican los anexos II y III del Reglamento (CE) núm. 396/2005 del Parlamento Europeo y del Consejo en lo relativo a los límites máximos de residuos de dietofencarb, mesotriona, metosulam y pirimifós-metilo en determinados productos.

- Reglamento (UE) 2016/60 de la Comisión, de 19 de enero de 2016, que modifica los anexos II y III del Reglamento (CE) núm. 396/2005 del Parlamento Europeo y del Consejo por lo que respecta a los límites máximos de residuos de clorpirifós en determinados productos.

- Reglamento (UE) 2016/67 de la Comisión, de 19 de enero de 2016, que modifica los anexos II, III y V del Reglamento (CE) núm. 396/2005 del Parlamento Europeo y del Consejo en lo relativo a los límites máximos de residuos de ametoctradina, clorotalonil, difenilamina, flonicamid, fluazinam, fluoxastrobina, halauxifen-metilo, propamocarb, protioconazol, tiacloprid y trifloxistrobina en determinados productos.

- Reglamento (UE) 2016/75 de la Comisión, de 21 de enero de 2016, que modifica el anexo III del Reglamento (CE) núm. 396/2005 del Parlamento Europeo y del Consejo por lo que respecta a los límites máximos de residuos de fosetil en determinados productos.

- Reglamento (UE) 2016/71 de la Comisión, de 26 de enero de 2016, que modifica los anexos II, III y V del Reglamento (CE) núm. 396/2005 del Parlamento Europeo y del Consejo en lo relativo a los límites máximos de residuos de las sustancias 1metilciclopropeno, flonicamid, flutriafol, ácido indolilacético, ácido indolilbutírico, petoxamida, pirimicarb, protioconazol y teflubenzurón en determinados productos.

- Reglamento (UE) 2016/156 de la Comisión, de 18 de enero de 2016, por el que se modifican los anexos II y III del Reglamento (CE) núm. 396/2005 del Parlamento Europeo y del Consejo por lo que respecta a los límites máximos de residuos de boscalida, clotianidina, tiametoxam, folpet y tolclofós-metilo en determinados productos.

Para terminar, y en relación con las disposiciones pesqueras que prohíben la pesca de determinadas especies en aguas internacionales y de la Unión Europea, se han dictado los siguientes reglamentos: 
- Reglamento (UE) 2015/1879 de la Comisión, de 15 de octubre de 2015, por el que se prohíbe la pesca del merlán en la zona VIII por parte de los buques que enarbolan pabellón de Bélgica.

- Reglamento (UE) 2015/1880 de la Comisión, de 15 de octubre de 2015, por el que se prohíbe la pesca del lenguado en las zonas VIIIa y VIIIb por parte de los buques que enarbolan pabellón de Bélgica.

- Reglamento (UE) 2015/1881 de la Comisión, de 15 de octubre de 2015, por el que se prohíbe la pesca del lenguado en las zonas VIIf y VIIg por parte de los buques que enarbolan pabellón de Bélgica.

- Reglamento (UE) 2015/1882 de la Comisión, de 15 de octubre de 2015, por el que se prohíbe la pesca de solla en zonas VIII, IX y X; en aguas de la Unión del CPACO 34.1.1 por parte de los buques que enarbolan pabellón de Bélgica.

- Reglamento (UE) 2015/1883 de la Comisión, de 15 de octubre de 2015, por el que se prohíbe la pesca de rayas en aguas de la Unión de las zonas VIII y IX por parte de los buques que enarbolan pabellón de Bélgica.

- Reglamento (UE) 2015/1895 de la Comisión, de 16 de octubre de 2015, por el que se prohíbe la pesca de rapes en las zonas VIIIa, VIIIb, VIIId y VIIIe por parte de los buques que enarbolan pabellón de Bélgica.

- Reglamento (UE) 2015/1896 de la Comisión, de 16 de octubre de 2015, por el que se prohíbe la pesca de merluza en las zonas VIIIa, VIIIb, VIIId y VIIIe por parte de los buques que enarbolan pabellón de Bélgica.

- Reglamento (UE) 2015/1903 de la Comisión, de 20 de octubre de 2015, por el que se prohíbe la pesca de cigalas en las zonas VIIIa, VIIIb, VIIId y VIIIe por parte de los buques que enarbolan pabellón de Bélgica.

- Reglamento (UE) 2015/1904 de la Comisión, de 20 de octubre de 2015, por el que se prohíbe la pesca de gallos en las zonas VIIIa, VIIIb, VIIId y VIIIe por parte de los buques que enarbolan pabellón de Bélgica.

- Reglamento (UE) 2015/1950 de la Comisión, de 26 de octubre de 2015, por el que se prohíbe la pesca de merlán en las zonas VI; aguas de la Unión y aguas internacionales de la zona $\mathrm{Vb}$; aguas internacionales de las zonas XII y XIV por parte de los buques que enarbolan pabellón de Irlanda. 
- Reglamento (UE) 2015/1995 de la Comisión, de 3 de noviembre de 2015, por el que se prohíbe la pesca de bacalao en la zona del Kattegat por parte de los buques que enarbolan pabellón de Suecia.

- Reglamento (UE) 2015/2073 de la Comisión, de 16 de noviembre de 2015, por el que se prohíbe la pesca de bacalao en la zona IV, en aguas de la Unión de la zona IIa y en la parte de IIIa no incluida en el Skagerrak y el Kattegat por parte de los buques que enarbolan pabellón de Suecia.

- Reglamento (UE) 2015/2074 de la Comisión, de 16 de noviembre de 2015, por el que se prohíbe la pesca de gamba nórdica en aguas de Noruega al sur del paralelo $62^{\circ} \mathrm{N}$ por parte de los buques que enarbolan pabellón de Suecia.

- Reglamento (UE) 2015/2092 de la Comisión, de 17 de noviembre de 2015, por el que se prohíbe la pesca de bacalao en aguas de Noruega al sur del paralelo $62^{\circ} \mathrm{N}$ por parte de los buques que enarbolan pabellón de Suecia.

- Reglamento (UE) 2015/2104 de la Comisión, de 18 de noviembre de 2015, por el que se prohíbe la pesca de carbonero en aguas de Noruega al sur del paralelo $62^{\circ} \mathrm{N}$ por parte de los buques que enarbolan pabellón de Suecia.

- Reglamento (UE) 2015/2230 de la Comisión, de 30 de noviembre de 2015, por el que se prohíbe la pesca de brótola de fango en aguas de la UE y aguas internacionales de las zonas VIII y IX por parte de los buques que enarbolan pabellón de España.

- Reglamento (UE) 2015/2241 de la Comisión, de 1 de diciembre de 2015, por el que se prohíbe la pesca de caballa en las zonas VIIIc, IX y X; en aguas de la Unión del CPACO 34.1.1 por parte de los buques que enarbolan pabellón de Portugal.

- Reglamento (UE) 2015/2242 de la Comisión, de 1 de diciembre de 2015, por el que se prohíbe la pesca de brótola de fango en aguas de la Unión y aguas internacionales de las zonas V, VI y VII por parte de los buques que enarbolan pabellón de España.

- Reglamento (UE) 2015/2243 de la Comisión, de 1 de diciembre de 2015, por el que se prohíbe la pesca de rayas en aguas de la Unión de las zonas VIa, VIb, VIIa-c y VIIe-k por parte de los buques que enarbolan pabellón de Bélgica. 
- Reglamento (UE) 2015/2269 de la Comisión, de 3 de diciembre de 2015, por el que se prohíbe la pesca de bacalao en la zona VIId por parte de los buques que enarbolan pabellón de Bélgica.

- Reglamento (UE) 2015/2270 de la Comisión, de 3 de diciembre de 2015, por el que se prohíbe la pesca de solla en las zonas VIIh, VIIj y VIIk por parte de los buques que enarbolan pabellón de Francia.

- Reglamento (UE) 2015/2271 de la Comisión, de 3 de diciembre de 2015, por el que se prohíbe la pesca de maruca en aguas de la Unión y aguas internacionales de la zona V por parte de los buques que enarbolan pabellón de Francia.

- Reglamento (UE) 2015/2293 de la Comisión, de 4 de diciembre de 2015, por el que se prohíbe la pesca de bacalao en la zona NAFO 3M por parte de los buques que enarbolan pabellón de Portugal.

- Reglamento (UE) 2015/2339 de la Comisión, de 11 de diciembre de 2015, por el que se prohíbe la pesca de fletán negro en las zonas NAFO 3LMNO por parte de los buques que enarbolan pabellón de España.

\section{B. Reglamentos de ejecución}

El Reglamento (CE) núm. 1007/2009 autoriza, en determinadas condiciones, la comercialización de productos derivados de la foca procedentes de la caza realizada por los inuit u otras comunidades indígenas. También permite la comercialización de productos derivados de la foca cuando la importación de tales productos es de carácter ocasional y los productos importados consisten exclusivamente en objetos destinados al uso personal de los viajeros o de sus familiares. Sin embargo, con el fin de mejorar este reglamento es conveniente disponer que los organismos que cumplen determinados requisitos se incluyan en una lista de organismos reconocidos que emiten certificados del cumplimiento de las condiciones de comercialización de productos derivados de la foca. Asimismo, deben definirse unos modelos de certificado y sus copias para facilitar la gestión y comprobación de los certificados. Por todo ello, se dicta el Reglamento de Ejecución (UE) 2015/1850 de la Comisión, de 13 de octubre de 2015, por el que se establecen disposiciones de aplicación del Reglamento (CE) núm. 1007/2009 del Parlamento Europeo y del Consejo, sobre el comercio de productos derivados de la foca. 
El Reglamento (UE) núm. 165/2011 establece, además, también debido a la sobrepesca practicada en 2010, que la cuota de pesca de caballa asignada a España para 2015 y, en su caso, para años posteriores debe ser objeto de una reducción de 9.747 toneladas.

El 30 de marzo de 2015 las autoridades españolas informaron a la Comisión de que su cuota de pesca de caballa para 2014 no se había utilizado íntegramente y le solicitaron que tuviera en cuenta las toneladas no utilizadas a efectos de disminuir las reducciones previstas en dicho Reglamento. Los informes revelaron que la cuota española de pesca de caballa para 2014 no utilizada asciende a 5.652,891 toneladas. En consecuencia, se dicta el Reglamento de Ejecución (UE) 2015/1999 de la Comisión, de 9 de noviembre de 2015, que modifica el Reglamento (UE) núm. 165/2011 por el que se reducen determinadas cuotas de caballa asignadas a España para 2011 y años siguientes debido a la sobrepesca practicada en 2010 .

De conformidad con el artículo 105, apartado 1, del Reglamento (CE) núm. 1224/2009, si la Comisión establece que un Estado miembro ha rebasado las cuotas de pesca que se le hubieran asignado, se efectuarán deducciones de las futuras cuotas de pesca de dicho Estado miembro. En este contexto, a petición de Portugal, la gallineta nórdica en aguas de Noruega de diferentes zonas debe utilizarse como población alternativa a efectos de deducción de la sobrepesca del eglefino y carbonero en aguas de Noruega de dichas zonas. No obstante, la cuota portuguesa de 2015 de gallineta nórdica en aguas de Noruega de esas zonas no es suficiente para cubrir las deducciones debidas por ambas poblaciones sobreexplotadas, puesto que esta cuota debe utilizarse para toda la cantidad disponible. Por lo tanto, en el año o años siguientes deberá deducirse, de las capturas de carbonero en la misma zona, una cantidad restante de $145.616 \mathrm{~kg}$ hasta la plena restitución de la cantidad rebasada. Asimismo, una cantidad de $3.369 \mathrm{~kg}$, correspondiente al $10 \%$ de la cuota española adaptada de 2014 para cigala en determinadas zonas en aguas de la Unión, ya no está a disposición de España a raíz de una transferencia de cuota. Por consiguiente, la cantidad disponible de $9.287 \mathrm{~kg}$ restada de las deducciones pendientes de $19.000 \mathrm{~kg}$ relativas a esta población debe reducirse a $5.918 \mathrm{~kg}$ y una deducción adicional de $3.369 \mathrm{~kg}$ debe ser inmediatamente aplicable. Con el fin de reflejar dichas modificaciones, se adopta el Reglamento de Ejecución (UE) 2015/2404 de la Comisión, de 16 de diciembre de 2015, por el que se efectúan deducciones de las cuotas de pesca disponibles para determinadas poblaciones en 2015 
a causa de la sobrepesca practicada en años anteriores en otras poblaciones y por el que se modifica el Reglamento (UE) 2015/1801.

Dado que en la Unión ya no se fabrican, comercializan ni usan sellantes para diques con parafinas cloradas de cadena corta (PCCC) y que existen alternativas adecuadas al uso de esta sustancia en casi todas sus aplicaciones, la Comisión prohíbe su uso mediante la aprobación del Reglamento (UE) 2015/2030 de la Comisión, de 13 de noviembre de 2015, por el que se modifica el Reglamento (CE) núm. 850/2004 del Parlamento Europeo y del Consejo, sobre contaminantes orgánicos persistentes, con respecto al anexo I. No obstante, cabe advertir que esta prohibición no es aplicable a las cintas transportadoras utilizadas en el sector minero ni a los sellantes para diques que ya estaban en uso antes o en la fecha de entrada en vigor del presente reglamento. Asimismo, se autoriza la comercialización y el uso de artículos que contengan PCCC en concentraciones inferiores al $0,15 \%$ en peso.

Además, en relación con los reglamentos de ejecución podemos destacar especialmente las autorizaciones relativas al uso de diferentes sustancias como aditivo en piensos para todas las especies animales y, en menor medida, para su uso en biocidas. El Reglamento (CE) núm. 1831/2003 regula el uso de aditivos en la alimentación animal y establece los motivos y procedimientos para su autorización. Se han presentado numerosas solicitudes de autorización de diferentes productos como aditivo en piensos, ámbito que ha sido modificado recientemente por el Reglamento (UE) 2015/2294 de la Comisión, de 9 de diciembre de 2015, por el que se modifica el Reglamento (CE) núm. 1831/2003 del Parlamento Europeo y del Consejo para establecer un nuevo grupo funcional de aditivos para piensos, que añade un nuevo grupo funcional en la categoría de los "aditivos tecnológicos" para aquellos aditivos potenciadores de las condiciones higiénicas para mejorar la calidad de los piensos, lo que supone garantías adicionales de protección de la salud pública y animal.

La Autoridad Europea de Seguridad Alimentaria concluyó que, en las condiciones de uso propuestas, estos productos no tienen efectos adversos para la salud animal, la salud humana ni el medio ambiente, y que pueden considerarse eficaces en lo que se refiere a su contribución para cumplir los requisitos de contenido en la alimentación de las especies animales. Por consiguiente, procede autorizar el uso de dichas sustancias según lo especificado en sus anexos. En esta materia se han dictado los siguientes actos: 
- Reglamento de Ejecución (UE) 2015/2306 de la Comisión, de 10 de diciembre de 2015, relativo a la autorización del clorhidrato monohidrato de L-cisteína como aditivo en los piensos para gatos y perros.

- Reglamento de Ejecución (UE) 2015/2307 de la Comisión, de 10 de diciembre de 2015, relativo a la autorización del bisulfito sódico de menadiona y del bisulfito de menadiona nicotinamida como aditivos en piensos para todas las especies animales.

- Reglamento de Ejecución (UE) 2015/2304 de la Comisión, de 10 de diciembre de 2015, relativo a la autorización de un preparado de endo-1,4-beta-xilanasa y de endo1,3(4)-beta-glucanasa producidas por Talaromyces versatilis sp. nov. (IMI CC 378536) y por Talaromyces versatilis sp. nov. (DSM 26702) como aditivo para la alimentación animal de pavos de engorde y pavos destinados a la reproducción (titular de la autorización, Adisseo France S.A.S.).

- Reglamento de Ejecución (UE) 2015/2305 de la Comisión, de 10 de diciembre de 2015, relativo a la autorización del preparado de endo-1,4-beta-glucanasa (EC 3.2.1.4) producido por Trichoderma citrinoviride Bisset (IM SD142) como aditivo en piensos para pollos de engorde, especies menores de aves de corral de engorde y lechones destetados, y por el que se modifican los Reglamentos (CE) núm. 2148/2004 y (CE) no 1520/2007 (titular de la autorización, Huvepharma NV).

- Reglamento de Ejecución (UE) 2015/2382 de la Comisión, de 17 de diciembre de 2015, relativo a la autorización del preparado de $\alpha$-galactosidasa (EC 3.2.1.22) producida por Saccharomyces cerevisiae (CBS 615.94) y endo-1,4- $\beta$-glucanasa (EC 3.2.1.4) producida por Aspergillus niger (CBS 120604) como aditivo alimentario para gallinas ponedoras y especies menores de aves de corral ponedoras (titular de la autorización: Kerry Ingredients and Flavours).

En esta materia cabe igualmente destacar el Reglamento de Ejecución (UE) 2015/2383 de la Comisión, de 17 de diciembre de 2015, que modifica el anexo I del Reglamento (CE) núm. 669/2009 por lo que respecta a la lista de piensos y alimentos de origen no animal cuya importación está sujeta a controles oficiales más intensos, así como el Reglamento de Ejecución (UE) 2016/6 de la Comisión, de 5 de enero de 2016, por el que se imponen condiciones especiales a la importación de piensos y alimentos originarios o procedentes de Japón a raíz del accidente en la central nuclear de Fukushima y se deroga el Reglamento de Ejecución (UE) núm. 322/2014. 
Por otro lado, la Comisión ha dictado los siguientes actos en relación con la aprobación de determinadas sustancias activas contenidas en biocidas:

- Reglamento de Ejecución (UE) 2015/1981 de la Comisión, de 4 de noviembre de 2015, por el que se aprueba el uso del formaldehído liberado de la N,N-metilenbismorfolina como sustancia activa existente en biocidas de los tipos de productos 6 y 13.

- Reglamento de Ejecución (UE) 2015/1982 de la Comisión, de 4 de noviembre de 2015, por el que se aprueba el uso de hexaflumurón como sustancia activa existente en biocidas del tipo de producto 18 .

- Reglamento de Ejecución (UE) 2016/105 de la Comisión, de 27 de enero de 2016, por el que se aprueba el uso de bifenil-2-ol como sustancia activa existente en biocidas de los tipos de producto 1, 2, 4, 6 y 13.

- Reglamento de Ejecución (UE) 2016/124 de la Comisión, de 29 de enero de 2016, por el que se aprueba el uso del PHMB $(1600 ; 1.8)$ como sustancia activa existente en biocidas del tipo de producto 4 .

- Reglamento de Ejecución (UE) 2016/125 de la Comisión, de 29 de enero de 2016, por el que se aprueba el uso de PHMB $(1600 ; 1.8)$ como sustancia activa existente en biocidas de los tipos de producto 2, 3 y 11 .

- Reglamento de Ejecución (UE) 2016/131 de la Comisión, de 1 de febrero de 2016, por el que se aprueba la C(M)IT/MIT (3:1) como sustancia activa existente para su uso en biocidas de los tipos de producto 2, 4, 6, 11, 12 y 13.

\section{Reglamentos delegados}

La Conferencia de las Partes en la Convención Marco de las Naciones Unidas sobre el Cambio Climático en calidad de reunión de las Partes en el Protocolo de Kioto aprobó la enmienda de Doha, por la que se establece un segundo período de compromiso del Protocolo de Kioto, que empezó el 1 de enero de 2013 y finalizará el 31 de diciembre de 2020. Esta enmienda implica que la Unión Europea y los Estados miembros deberán consignar en sus respectivos registros del Protocolo de Kioto las nuevas unidades de cantidad atribuida de conformidad con la Decisión (UE) 2015/1339 del Consejo. 
En este sentido, dada la necesidad de realizar transferencias transitorias antes de que finalice el período adicional previsto para cumplir los compromisos del primer período del Protocolo de Kioto, se adopta el Reglamento Delegado (UE) 2015/1844 de la Comisión, de 13 de julio de 2015, por el que se modifica el Reglamento (UE) núm. 389/2013 en lo relativo a la ejecución técnica del Protocolo de Kioto después de 2012, el cual deberá entrar en vigor a la mayor brevedad posible.

\section{Decisiones de ejecución}

En virtud del Acuerdo entre la Comunidad Europea y Nueva Zelanda sobre medidas sanitarias aplicables al comercio de animales vivos y de productos animales, se establecen las medidas de salud pública y sanidad animal para los animales vivos y los productos de origen animal procedentes de Nueva Zelanda cuya equivalencia ha sido reconocida. Los envíos de animales vivos o productos de origen animal para los que se ha reconocido la equivalencia con respecto a las medidas de sanidad animal o a las medidas de salud pública, pero no con respecto a ambas, pueden importarse en la Unión siempre que las declaraciones adicionales aplicables de no equivalencia de los certificados veterinarios establecidos de conformidad con los modelos previstos en la legislación pertinente de la Unión sobre certificados veterinarios de importación figuren en el modelo de certificado sanitario.

En este sentido, procede enumerar dichos animales vivos y productos de origen animal en un anexo, y para ello se adopta la Decisión de Ejecución (UE) 2015/1901 de la Comisión, de 20 de octubre de 2015, por la que se establecen normas de certificación y un modelo de certificado sanitario para la importación en la Unión de envíos de animales vivos y de productos de origen animal procedentes de Nueva Zelanda y por la que se deroga la Decisión 2003/56/CE.

En relación con otra materia, después de que Italia notificase la presencia del pequeño escarabajo de la colmena (Aethina tumida) en las regiones de Calabria y Sicilia a finales de 2015, las normas internacionales establecidas por la Organización Mundial de Sanidad Animal (OIE) recomiendan que se realicen estudios anuales antes de que una zona pueda recuperar su estatus de zona libre de infestación. Es difícil establecer con exactitud la evolución epidemiológica de las infestaciones por el pequeño escarabajo de la colmena dado que las señales pueden pasar desapercibidas y que también pueden 
resultar infestadas las colonias de abejas silvestres. Las infestaciones notificadas previamente en Italia en 2014 fueron detectadas durante el otoño, entre septiembre y diciembre. Por lo tanto, el estudio anual en curso y el próximo estudio anual deberán cubrir todo el período otoñal de 2015 y 2016, respectivamente.

Teniendo en cuenta que aún no se ha establecido la situación epidemiológica en estas regiones de Italia y que no se dispone de más información sobre el estudio en curso y sobre el estudio próximo, es necesario incluso prolongar unos meses la aplicación de las medidas establecidas en la Decisión 2014/909/UE tras el fin previsto de la próxima campaña apícola a finales de noviembre de 2016. Procede, por lo tanto, modificar la Decisión de Ejecución 2014/909/UE, y, en consecuencia, se adopta la Decisión de Ejecución (UE) 2015/1943 de la Comisión, de 27 de octubre de 2015, por la que se modifica la Decisión de Ejecución 2014/909/UE ampliando el período de aplicación de las medidas de protección en relación con el pequeño escarabajo de la colmena en Italia. Atendiendo a otro tema, se ha de poner de relieve que la caballa y el arenque son especies migratorias de amplia distribución. Con el fin de armonizar los procedimientos de control e inspección de la pesca de caballa y arenque en las aguas adyacentes a las aguas occidentales, es conveniente, por una parte, incluir divisiones en el programa específico de control e inspección, $y$, por otra, mantener el programa específico de control e inspección hasta el 31 de diciembre de 2018 para garantizar unas condiciones equitativas durante la introducción de la obligación de desembarque.

En este sentido, la Comisión, tras realizar las consultas previstas sobre la gestión de la caballa en 2015 en el programa específico de control e inspección en el mar Báltico y en el mar del Norte, se encuentra con los informes emitidos por Noruega y las Islas Feroe, que señalan que los desembarques de arenque, caballa y jurel se han reducido significativamente, ya que a partir de ahora se basarán en la evaluación de riesgos, y su aplicación se ha ampliado a los desembarques de bacaladilla. Por lo tanto, procede modificar en consecuencia los parámetros de referencia del anexo II de la Decisión de Ejecución 2012/807/CEE. Por ello, se dicta la Decisión (UE) 2015/1944 de Ejecución de la Comisión, de 28 de octubre de 2015, que modifica la Decisión de Ejecución 2012/807/UE de la Comisión por la que se establece un programa específico de control e inspección de las pesquerías pelágicas en las aguas occidentales del Atlántico Nororiental. 
En otro orden de consideraciones, el 10 de marzo de 2015 el proveedor DENSO Corporation presentó una solicitud de aprobación de su segunda tecnología innovadora: el alternador de alta eficiencia DENSO para la clase de salida de 100 A a 250 A. La solicitud ha sido evaluada de conformidad con la normativa en la materia y con las orientaciones técnicas.

El solicitante ha demostrado que la utilización de un alternador de alta eficiencia del tipo descrito en esta solicitud no superó el 3\% de los turismos nuevos matriculados en el año de referencia (2009). Asimismo, la Comisión considera que el solicitante ha demostrado satisfactoriamente que la reducción de emisiones lograda gracias a la tecnología innovadora es de al menos $1 \mathrm{~g}$ de $\mathrm{CO}_{2} / \mathrm{km}$. Por lo tanto, considera que no deben plantearse objeciones a la aprobación de la tecnología innovadora en cuestión y, por ello, aprueba la Decisión de Ejecución (UE) 2015/2280 de la Comisión, de 7 de diciembre de 2015, relativa a la aprobación del alternador de alta eficiencia DENSO como tecnología innovadora para la reducción de las emisiones de $\mathrm{CO}_{2}$ de los turismos de conformidad con el Reglamento (CE) núm. 443/2009 del Parlamento Europeo y del Consejo.

La Comisión debe determinar los límites de sustancias reguladas importadas y asignar cuotas a empresas. Asimismo, debe determinar las cantidades de sustancias reguladas distintas de los hidroclorofluorocarburos que pueden utilizarse para usos esenciales de laboratorio y análisis y las empresas que pueden utilizarlas. La Comisión publicó un anuncio dirigido a las empresas que tenían la intención, para 2016, de importar en la Unión Europea, o exportar desde esta, sustancias reguladas que agotan la capa de ozono y a las empresas que tenían la intención de solicitar una cuota para 2016 con objeto de utilizar dichas sustancias para usos analíticos y de laboratorio, y, en respuesta, recibió declaraciones sobre las importaciones previstas para 2016. Teniendo en cuenta toda la información recabada por la Comisión en esta materia, se dicta la Decisión de Ejecución (UE) 2015/2337 de la Comisión, de 11 de diciembre de 2015, por la que se determinan los límites cuantitativos y se asignan cuotas de sustancias reguladas en el marco del Reglamento (CE) núm. 1005/2009 del Parlamento Europeo y del Consejo, sobre las sustancias que agotan la capa de ozono, para el período comprendido entre el 1 de enero y el 31 de diciembre de 2016.

En otro ámbito, el 27 de mayo de 2015 el proveedor Mitsubishi Electric Corporation (MELCO), representado en la Unión por Mitsubishi Electric Automotive Europe B.V., 
presentó una solicitud de aprobación de su segunda tecnología innovadora: el motogenerador MELCO. El motogenerador MELCO tiene una función de generador similar a la de un alternador normal. Comparado con un alternador de referencia, reduce la pérdida en el cobre del estátor mediante un estátor con factor de forma ultra alto fabricado según un método de bobinado de alambre de densidad ultra alta y una nueva estructura de refrigeración bidireccional. También reduce la pérdida en el hierro del estátor mediante un núcleo del estátor de acero electromagnético fino y de alto grado. Por último, reduce la pérdida de rectificación mediante un nuevo módulo de transistores de efecto de campo con óxido de metal semiconductor.

En este sentido, la Comisión considera que el solicitante ha demostrado satisfactoriamente que la reducción de emisiones lograda gracias a la tecnología innovadora es de al menos $1 \mathrm{~g}$ de $\mathrm{CO}_{2} / \mathrm{km}$. Por lo tanto, considera que no deben plantearse objeciones a la aprobación de la tecnología innovadora en cuestión y por ello aprueba la Decisión de Ejecución (UE) 2016/265 de la Comisión, de 25 de febrero de 2016, relativa a la aprobación del motogenerador MELCO como tecnología innovadora para la reducción de las emisiones de $\mathrm{CO}_{2}$ de los turismos de conformidad con el Reglamento (CE) núm. 443/2009 del Parlamento Europeo y del Consejo.

Asimismo, en materia de sustancias activas utilizadas en biocidas, se han adoptado las siguientes decisiones de ejecución:

- Decisión de Ejecución (UE) 2016/107 de la Comisión, de 27 de enero de 2016, por la que no se aprueba la cibutrina como sustancia activa existente para su uso en biocidas del tipo de producto 21 .

- Decisión de Ejecución (UE) 2016/108 de la Comisión, de 27 de enero de 2016, por la que no se aprueba la 2-Butanona, peróxido como sustancia activa existente para su uso en biocidas de los tipos de producto 1 y 2.

— Decisión de Ejecución (UE) 2016/109 de la Comisión, de 27 de enero de 2016, por la que no se aprueba el uso del PHMB $(1600 ; 1,8)$ como sustancia activa existente en biocidas de los tipos de producto 1,6 y 9.

- Decisión de Ejecución (UE) 2016/110 de la Comisión, de 27 de enero de 2016, por la que no se aprueba el triclosán como sustancia activa existente para su uso en biocidas del tipo de producto 1 . 
Para terminar, debemos destacar como novedad las decisiones de ejecución adoptadas en relación con las listas de lugares de importancia comunitaria (también conocidos como LIC) creadas en virtud de la Directiva 92/43/CEE del Consejo, de 21 de mayo de 1992, relativa a la conservación de los hábitats naturales y de la fauna y flora silvestres. En este sentido, debemos señalar que los lugares incluidos en estas listas de lugares de importancia comunitaria de la región biogeográfica panónica forman parte de la red Natura 2000, que es un elemento esencial para la protección de la biodiversidad de la Unión. Dichos lugares son propuestos por los Estados miembros.

A fin de seguir avanzando en el establecimiento concreto de la red Natura 2000, y en el marco de la adaptación dinámica de dicha red, las listas de lugares de importancia comunitaria se revisan periódicamente. A tales efectos, se dictan las siguientes decisiones:

- Decisión de Ejecución (UE) 2015/2368 de la Comisión, de 26 de noviembre de 2015, por la que se adopta la séptima lista actualizada de lugares de importancia comunitaria de la región biogeográfica panónica [notificada con el número $\mathrm{C}(2015)$ 8189].

- Decisión de Ejecución (UE) 2015/2369 de la Comisión, de 26 de noviembre de 2015, por la que se adopta la novena lista actualizada de lugares de importancia comunitaria de la región biogeográfica continental [notificada con el número $\mathrm{C}(2015)$ 8191].

- Decisión de Ejecución (UE) 2015/2370 de la Comisión, de 26 de noviembre de 2015, por la que se adopta la novena lista actualizada de lugares de importancia comunitaria de la región biogeográfica alpina [notificada con el número C(2015) 8206].

- Decisión de Ejecución (UE) 2015/2371 de la Comisión, de 26 de noviembre de 2015, por la que se adopta la novena lista actualizada de lugares de importancia comunitaria de la región biogeográfica boreal [notificada con el número C(2015) 8214].

- Decisión de Ejecución (UE) 2015/2372 de la Comisión, de 26 de noviembre de 2015, por la que se adopta la quinta lista actualizada de lugares de importancia comunitaria de la región biogeográfica macaronésica [notificada con el número $C(2015)$ 8215].

- Decisión de Ejecución (UE) 2015/2373 de la Comisión, de 26 de noviembre de 2015, por la que se adopta la novena lista actualizada de lugares de importancia 
comunitaria de la región biogeográfica atlántica [notificada con el número $C(2015)$ 8219].

- Decisión de Ejecución (UE) 2015/2374 de la Comisión, de 26 de noviembre de 2015, por la que se adopta la novena lista actualizada de lugares de importancia comunitaria de la región biogeográfica mediterránea [notificada con el número C(2015) 8222].

- Decisión de Ejecución (UE) 2015/2375 de la Comisión, de 26 de noviembre de 2015, por la que se adopta la tercera lista actualizada de lugares de importancia comunitaria de la región biogeográfica del mar Negro [notificada con el número C(2015) 8226].

\section{E. Dictámenes y comunicaciones de la Comisión}

Entre los dictámenes dictados por la Comisión, cabe destacar:

- Dictamen de la Comisión, de 25 de noviembre de 2015, sobre el proyecto modificado de evacuación de los residuos radiactivos producto del plan de rehabilitación del emplazamiento de Dounreay, Caithness (Escocia, Reino Unido), mediante el cual se establece por la Comisión, tras recibir los informes y datos del Reino Unido según los cuales la ejecución del proyecto modificado de evacuación de los residuos radiactivos producto del plan de rehabilitación del emplazamiento de Dounreay, sea del tipo que sea y tanto en condiciones de funcionamiento normales como en caso de un accidente del tipo y magnitud considerados en los datos generales, que no es probable que dé lugar a una contaminación radiactiva del agua, el suelo o el espacio aéreo de otro Estado miembro que sea significativa desde el punto de vista sanitario, en relación con lo dispuesto en las nuevas normas básicas de seguridad (Directiva 2013/59/Euratom).

- Dictamen de la Comisión, de 4 de diciembre de 2015, sobre el plan de evacuación de residuos radiactivos producto de la clausura y el desmantelamiento de la planta de reelaboración de La Hague UP2-400, situada en Francia. Este dictamen señala que no es probable que la aplicación del plan de evacuación de los residuos radiactivos, del tipo que sea, producto de la clausura y el desmantelamiento de la planta de reelaboración de La Hague UP2-400 (Francia), tanto en caso de funcionamiento normal como en caso de accidente del tipo y la magnitud considerados en los datos generales, 
cause una contaminación radiactiva del agua, el suelo o el espacio aéreo de otro Estado miembro o país vecino que sea significativa desde el punto de vista sanitario, en relación con lo dispuesto en las nuevas normas básicas de seguridad (Directiva 2013/59/Euratom).

\subsection{Otros}

\section{A. Comité de las Regiones}

El Dictamen del Comité de las Regiones Europeo - Contribución al control de la adecuación de la Directiva relativa a la conservación de las aves silvestres y la Directiva relativa a la conservación de los hábitats naturales y de la fauna y flora silvestres, (2016/C 051/10), subraya que en la actualidad uno de los mayores retos medioambientales, de aquí a 2020, consiste en frenar la pérdida de biodiversidad y de hábitats naturales, detener la degradación de los servicios ecosistémicos y fomentar su restauración.

A tales efectos, en este dictamen el Comité realiza algunas reflexiones y aportaciones para paliar esta pérdida y degradación. En primer lugar, lo más importante para el Comité es la falta de aplicación de las normas en materia de protección de especies y hábitats, esto es, las directivas de conservación de la naturaleza, tanto a escala local, como regional y nacional. Además, recuerda el papel clave que desempeñan los entes locales y regionales a la hora de aplicar las directivas de conservación de la naturaleza.

Por lo tanto, considera que el problema de fondo no radica en la regulación sobre esta materia, sino en su aplicación. En este sentido, manifiesta su preocupación por la destrucción de varios espacios Natura 2000 y por los niveles actuales de caza y captura ilegales de aves y otras especies animales, y está convencido de que es necesario realizar un mayor esfuerzo a todos los niveles de gobierno para supervisar y hacer cumplir los requisitos de las directivas de conservación de la naturaleza. Especialmente, que la Comisión Europea desempeñe su función de guardiana del derecho de la Unión, a la que pide, en este contexto, que tenga debidamente en cuenta las denuncias relativas a la aplicación del derecho de la Unión e incoe los procedimientos de infracción correspondientes. 


\section{B. Comité Económico y Social Europeo}

El 25 de marzo de 2015, de conformidad con el artículo 43, apartado 2, del Tratado de Funcionamiento de la Unión Europea, la Comisión Europea decidió consultar al Comité Económico y Social Europeo sobre el tema "El Protocolo de París, un plan rector para combatir el cambio climático más allá de 2020”. En este contexto, el Comité Económico y Social Europeo adopta el Dictamen sobre el tema "El Protocolo de París, un plan rector para combatir el cambio climático más allá de 2020" [COM(2015) 81 final] (2015/C 383/11), en el que apoya la posición presentada por la Comisión y confía en que las partes negociadoras de la COP 21 concluyan finalmente un acuerdo ambicioso y equitativo de carácter vinculante. Entiende que es de vital importancia que todas las partes de la Convención Marco de las Naciones Unidas sobre el Cambio Climático, sin excepción, asuman su responsabilidad para alcanzar el verdadero objetivo, a saber, la estabilización de las concentraciones de gases de efecto invernadero en la atmósfera a un nivel que impida interferencias antropogénicas peligrosas en el sistema climático. Solo de este modo podrán evitarse daños incluso mayores para el ser humano, el medio ambiente y las generaciones futuras. Para ello, considera que el principio de la responsabilidad común pero diferenciada es correcto.

Asimismo, el Comité estima que lo que puede salvar el clima no serán los ambiciosos resultados de la COP 21, sino sobre todo su aplicación coherente. Para una aplicación coherente entiende que se debe tener en consideración que finalmente estas medidas no serán aplicadas por los políticos, sino por los ciudadanos. Esta es la crítica más importante realizada por el Comité y en la que insiste varias veces en el Dictamen que nos ocupa, basada en el hecho de que la UE siga sin comprender del todo el papel central que la sociedad civil debe desempeñar en este proceso.

Pasando a otra cuestión, el 30 de abril de 2015, el Parlamento Europeo y, el 7 de mayo de 2015, el Consejo decidieron, de conformidad con el artículo 43, apartado 2, del Tratado de Funcionamiento de la Unión Europea, consultar al Comité Económico y Social Europeo sobre la Propuesta de Reglamento del Parlamento Europeo y del Consejo relativo a un plan de recuperación plurianual para el atún rojo del Atlántico oriental y el Mediterráneo y por el que se deroga el Reglamento (CE) núm. 302/2009. Entendiendo que el contenido de la propuesta es satisfactorio y $\sin$ ser objeto de ninguna otra observación, el Comité dicta el Dictamen sobre la Propuesta de Reglamento del Parlamento Europeo y del Consejo relativo a un plan de recuperación 
plurianual para el atún rojo del Atlántico oriental y el Mediterráneo y por el que se deroga el Reglamento (CE) núm. 302/2009, [COM(2015) 180 final — 2015/0096 COD], (2015/C 383/15).

En otra materia, se aprueba el Dictamen del Comité Económico y Social Europeo sobre el tema "La agricultura, las zonas rurales y el desarrollo sostenible en los países de la Asociación Oriental" (2016/C 013/20). En el marco medioambiental, el Dictamen apuesta por una modernización estratégica del sector agrícola en los países de la Asociación Oriental que aumente la producción nacional de alimentos seguros de manera sostenible, para lo cual concede un gran valor a la aplicación del Programa Europeo de Vecindad para la Agricultura y el Desarrollo Rural (ENPARD), así como a compartir la experiencia y los conocimientos de la Unión en estas materias. Esto no solo contribuirá a la creación de empleo, sino también a mejorar la calidad de vida de los habitantes de las zonas rurales.

El Dictamen del Comité Económico y Social Europeo sobre "Las alegaciones medioambientales, sociales y de propiedades saludables en el mercado interior" (2015/C 383/02) versa sobre la eficacia medioambiental y social de los productos y servicios comercializados en la Unión Europea. El Comité considera importante que la Comisión proponga métodos europeos para evaluar y comunicar el impacto ambiental global de los productos y servicios que consumimos habitualmente. Igualmente, se debería elaborar un inventario de etiquetas oficiales, especificando su significado y las entidades acreditadas para su otorgamiento, validez y contraste. En todo caso, los consumidores tienen derecho a disponer de una información clara y precisa sobre el origen de los productos y su impacto medioambiental.

Asimismo, el CESE espera que la Comisión revise, como prolongación de la Directiva 2005/29/CE, las directrices para facilitar el acceso por parte de consumidores y empresas a una información fiable y transparente que clarifique la aplicación de las alegaciones medioambientales, éticas y de salud en la comunicación comercial. Es cierto que en la Unión Europea ya existe un elevado número de empresas que ofrecen al consumidor información completa, veraz y transparente sobre las características esenciales de los bienes y servicios puestos a disposición en el mercado. No obstante, mediante la utilización inadecuada de alegaciones medioambientales, sociales y de salud, se está perjudicando a las empresas que cumplen la normativa y a los consumidores que adquieren los bienes y servicios. Por ello, se debe adoptar un marco 
jurídico íntegro para las comunicaciones comerciales en este contexto, pues el CESE considera que el actual está incompleto y es inútilmente complejo, inconsistente y, a veces, incoherente.

La industria de alta mar comprende la producción de energía y los sectores de la extracción marina de gas, petróleo y minerales. El CESE sostiene que los mercados relacionados con estos sectores evolucionan de forma especialmente dinámica y ofrecen oportunidades de desarrollar un crecimiento inteligente, sostenible y a largo plazo. Por ello, se aprueba el Dictamen del Comité Económico y Social Europeo sobre el tema "Perspectivas de desarrollo inteligente y sostenible a largo plazo de la industria europea de alta mar y su relación con los sectores marítimos de la UE" (2016/C 013/12), donde señala que, para garantizar un desarrollo sostenible de la industria de alta mar, es necesario establecer un marco coherente y una visión estratégica europea que garanticen el uso de tecnologías de vanguardia y soluciones innovadoras para este sector.

Tras realizar un análisis exhaustivo de las relaciones entre la industria de alta mar y la industria marítima europea, el Comité constata que de la industria de alta mar surgen impulsos muy potentes, y a menudo "ecológicos", para la industria marítima europea, en particular los sectores de la construcción naval, la reparación de buques y los equipos marinos.

Asimismo, entiende que debería elaborarse y aplicarse un programa de "cultura de la seguridad" y que habría que adoptar medidas destinadas a promover y respaldar la acreditación de los centros de formación por parte de las empresas del sector de alta mar con vistas a fomentar y apoyar una formación más exhaustiva en cuestiones de seguridad y de protección del medio ambiente en este sector.

Por último, considera que los procedimientos para la perforación horizontal y la fracturación hidráulica en el mar deberían adaptarse a las normas de protección medioambiental. 